\title{
Atherosclerosis
}

\section{Wall shear stress estimated by 3D-QCA can predict cardiovascular events in lesions with borderline negative fractional flow reserve \\ --Manuscript Draft--}

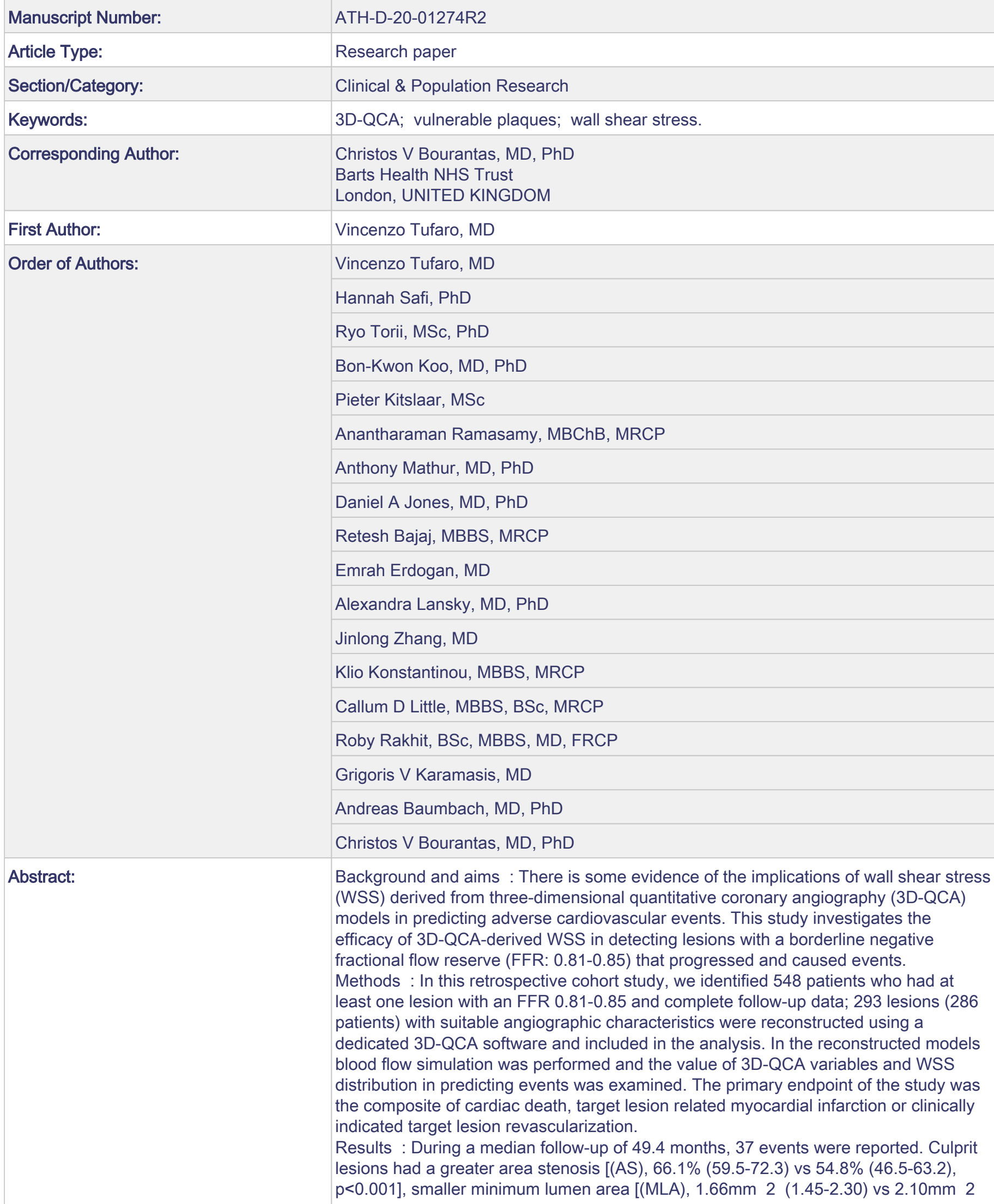


(1.69-2.70), $\mathrm{p}=0.011]$ and higher maximum WSS [9.0Pa (5.10-12.46) vs 5.0Pa (3.377.54), $p<0.001$ ] than those that remained quiescent. In multivariable analysis, AS [hazard ratio (HR): 1.06, 95\% confidence interval $(\mathrm{Cl}): 1.03-1.10, p=0.001$ ] and maximum WSS (HR: 1.08, 95\% Cl: 1.02-1.14, $\mathrm{p}=0.012$ ) were the only independent predictors of the primary endpoint. Lesions with an increased AS ( $\geq 58.6 \%)$ that were exposed to high WSS $(\geq 7.69 \mathrm{~Pa})$ were more likely to progress and cause events (27.8\%) than those with a low AS exposed to high WSS (7.4\%) or those exposed to low WSS that had increased $(12.8 \%)$ or low AS $(2.7 \%, p<0.001)$.

Conclusions : This study for the first time highlights the potential value of 3D-QCAderived WSS in detecting among lesions with a borderline negative FFR those that cause cardiovascular events. 
Lesions with fractional flow reserve between 0.81 and $0.85(n=293)$

AS<58.6\%

Max WSS<7.69Pa
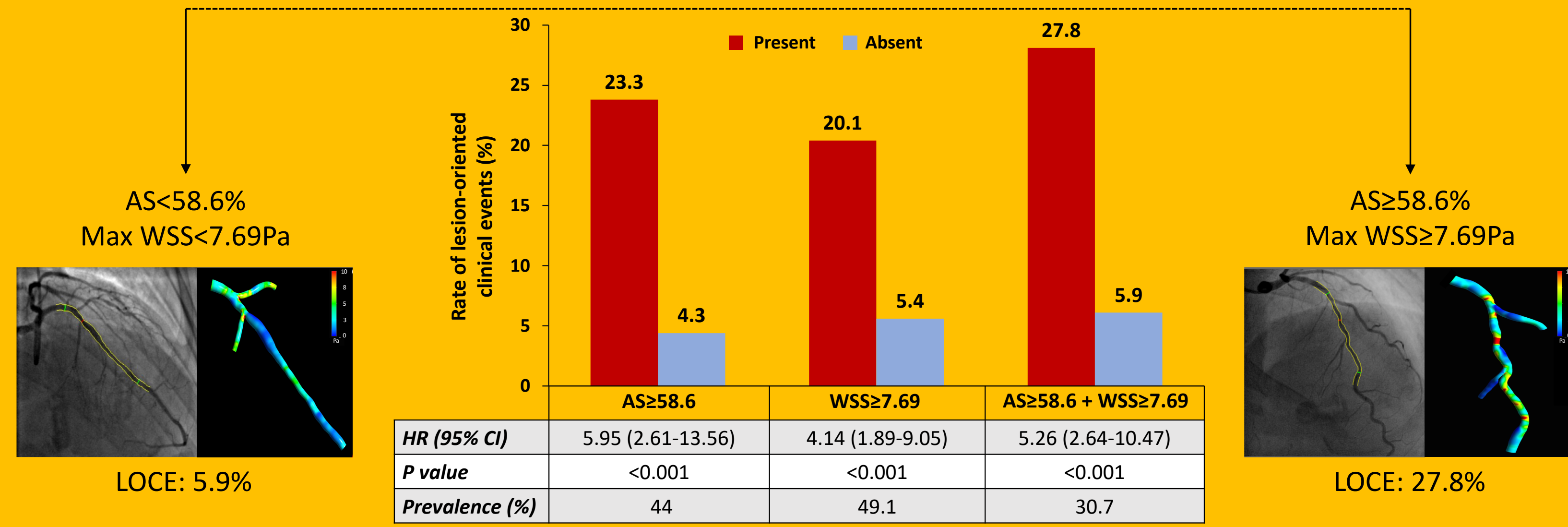

LOCE: $5.9 \%$

\begin{tabular}{|l|c|c|c|}
\hline P value & $<0.001$ & $<0.001$ & $<0.001$ \\
\hline Prevalence (\%) & 44 & 49.1 & 30.7 \\
\hline
\end{tabular}

LOCE: $27.8 \%$ 
3

4 Vincenzo Tufaro ${ }^{1,2 \#}$, MD; Hannah Safi ${ }^{1,3, \#, ~ P h D ; ~ R y o ~ T o r i i ~}{ }^{4}, \mathrm{MSc}, \mathrm{PhD}$; Bon-Kwon Koo ${ }^{5}$, MD, PhD;

5 Pieter Kitslaar ${ }^{6,7}, \mathrm{MSc}$; Anantharaman Ramasamy ${ }^{1,2}, \mathrm{MBChB}, \mathrm{MRCP} ;$ Anthony Mathur ${ }^{1,2}, \mathrm{MD}, \mathrm{PhD}$;

6 Daniel A. Jones ${ }^{1,2}, \mathrm{MD}, \mathrm{PhD}$; Retesh Bajaj ${ }^{1,2}$, MBBS, MRCP; Emrah Erdoğan, MD ${ }^{1,8}$; Alexandra

7 Lansky ${ }^{2,9}, \mathrm{MD}, \mathrm{PhD}$; Jinlong Zhang ${ }^{5}$, MD; Klio Konstantinou ${ }^{10}$, MBBS, MRCP; Callum D. Little ${ }^{11}$,

8 MBBS, BSc, MRCP; Roby Rakhit ${ }^{11}$, BSc, MBBS, MD, FRCP; Grigoris V. Karamasis ${ }^{10}$, MD; Andreas

9 Baumbach ${ }^{1,2,9}, \mathrm{MD}, \mathrm{PhD}$; Christos V. Bourantas ${ }^{1,2,3}, \mathrm{MD}, \mathrm{PhD}$

10

$11{ }^{1}$ Department of Cardiology, Barts Heart Centre, Barts Health NHS Trust, London, UK

$12{ }^{2}$ Centre for Cardiovascular Medicine and Devices, William Harvey Research Institute, Queen Mary

13 University London, UK

$14{ }^{3}$ Institute of Cardiovascular Sciences, University College London, London, UK
${ }^{4}$ Department of Mechanical Engineering, University College London, London, UK

${ }^{5}$ Department of Internal Medicine and Cardiovascular Centre, Seoul National University Hospital,
${ }^{6}$ Division of Image Processing, Department of Radiology, Leiden University Medical Centre, Leiden, The Netherlands.

${ }^{7}$ Medis Medical Imaging Systems bv, Leiden, The Netherlands

${ }^{8}$ Department of Cardiology, Van Yüzüncü Y1l University, Van, Turkey

${ }^{9}$ Yale University School of Medicine, New Haven, Connecticut, US

${ }^{10}$ Department of Cardiology, Essex Cardiothoracic Centre, Basildon, UK

${ }^{11}$ Department of Cardiology, Royal Free London NHS Trust, London, UK

\footnotetext{
\# The first two authors have contributed equally to this work
} 


\section{Corresponding author}

1

2

3

4

5

6

7

8

9
2 Christos Bourantas, $\mathrm{MD}, \mathrm{PhD}$

3 Consultant Cardiologist, Barts Heart Centre

4 West Smithfield, London EC1A 7BE

5 E-mail: cbourantas@gmail.com

6 Phone: +44 207377 7000, Fax: +44 2077919670

7

8 Word count: 4739.

9

10 


\section{1}

2 Background and aims: There is some evidence of the implications of wall shear stress (WSS) derived

3 from three-dimensional quantitative coronary angiography (3D-QCA) models in predicting adverse

4 cardiovascular events. This study investigates the efficacy of 3D-QCA-derived WSS in detecting lesions 5 with a borderline negative fractional flow reserve (FFR: 0.81-0.85) that progressed and caused events.

6 Methods: In this retrospective cohort study, we identified 548 patients who had at least one lesion with 7 an FFR 0.81-0.85 and complete follow-up data; 293 lesions (286 patients) with suitable angiographic 8 characteristics were reconstructed using a dedicated 3D-QCA software and included in the analysis. In 9 the reconstructed models blood flow simulation was performed and the value of 3D-QCA variables and WSS distribution in predicting events was examined. The primary endpoint of the study was the composite of cardiac death, target lesion related myocardial infarction or clinically indicated target lesion revascularization.

Results: During a median follow-up of 49.4 months, 37 events were reported. Culprit lesions had a greater area stenosis [(AS), 66.1\% (59.5-72.3) vs 54.8\% (46.5-63.2), $\mathrm{p}<0.001]$, smaller minimum lumen area [(MLA), $1.66 \mathrm{~mm}^{2}(1.45-2.30)$ vs $\left.2.10 \mathrm{~mm}^{2}(1.69-2.70), \mathrm{p}=0.011\right]$ and higher maximum WSS [9.0Pa $(5.10-12.46)$ vs $5.0 \mathrm{~Pa}(3.37-7.54), \mathrm{p}<0.001]$ than those that remained quiescent. In multivariable analysis, AS [hazard ratio (HR): 1.06, 95\% confidence interval (CI): 1.03-1.10, $\mathrm{p}=0.001$ ] and maximum WSS (HR: $1.08,95 \%$ CI: $1.02-1.14, \mathrm{p}=0.012$ ) were the only independent predictors of the primary endpoint. Lesions with an increased AS $(\geq 58.6 \%)$ that were exposed to high WSS $(\geq 7.69 \mathrm{~Pa})$ were more likely to progress and cause events $(27.8 \%)$ than those with a low AS exposed to high WSS $(7.4 \%)$ or those exposed to low WSS that had increased $(12.8 \%)$ or low $\operatorname{AS}(2.7 \%, \mathrm{p}<0.001)$.

Conclusions: This study for the first time highlights the potential value of 3D-QCA-derived WSS in detecting among lesions with a borderline negative FFR those that cause cardiovascular events.

Keywords: 3D-QCA, vulnerable plaques, wall shear stress. 


\section{INTRODUCTION}

2 Fractional flow reserve (FFR) is the current standard for the invasive assessment of lesion severity in

3 patients with intermediate stenoses and a cut-off of $\leq 0.80$ has been proposed to guide coronary 4 revascularisation. ${ }^{1}$ Nevertheless, patients with a borderline negative FFR of $0.81-0.85$ are at a high-risk 5 of suffering an event with studies showing a lesion related event rate of up to $30 \%$ at 4.5 years of follow6 up, which is much higher to the event rate reported in lesions with an FFR between $0.86-0.90$ or $>0.91$. $^{2-}$

$7 \quad{ }^{4}$ Moreover, lesions with an FFR 0.81-0.85 accounted for approximately one third of the lesions with a 8 negative FFR. ${ }^{2,5}$ Therefore, identification of new imaging and/or physiological markers that will enable 9 better risk stratification in these lesions is of utmost importance. Local haemodynamic forces and in particular wall shear stress (WSS) is a well-known instigator of 11 atherosclerosis since it promotes mechano-transduction pathways that regulate plaque formation and the development of vulnerable lesions. ${ }^{6}$ Recent reports have shown that WSS estimated in models reconstructed by intravascular imaging provides useful prognostic information and identification of non-flow limiting plaques that are prone to cause events. ${ }^{7,8}$ Despite the convincing evidence highlighting the predictive value of WSS, its application in the clinical practice to stratify cardiovascular risk is limited as intravascular imaging is not routinely used to assess lesion severity. In addition, intravascular imaging analysis, coronary reconstruction, blood flow simulation and WSS estimation are time consuming processes and require expertise that restrict their application in selected core labs with experience in the field. ${ }^{9}$

Three-dimensional quantitative coronary angiography (3D-QCA) modelling offers an attractive alternative for WSS computation as it enables reliable evaluation of lesion severity and real-time reconstruction of coronary artery anatomy. ${ }^{10}$ A recent report has shown that 3D-QCA derived WSS correlates well with the WSS estimated in models reconstructed by intravascular imaging data, ${ }^{11}$ while two reports have highlighted the potential value of 3D-QCA-based hemodynamic modelling in detecting amongst obstructive lesions or lesions with a vulnerable phenotype those that caused events. ${ }^{12,13}$ In this study, we examine for the first time the role of 3D-QCA-derived WSS in identifying vulnerable plaques and stratifying cardiovascular risk in patients with a borderline negative FFR who did not have an invasive assessment of lesion morphology. 


\section{Studied patients}

3 Patients who underwent a coronary angiography from January 2012 to June 2017 at three Cardiology

4 Departments in the United Kingdom (Barts Heart Centre, London; Essex Cardiothoracic Centre, 5 Basildon; Royal Free Hospital, London), that had at least one intermediate lesion with a borderline 6 negative FFR (FFR: 0.81-0.85) and did not have revascularization in this lesion were considered for 7 inclusion. The FFR protocol is described in detail in the Online Supplementary Material.

8 The present analysis included only patients with complete follow-up data (until the $1^{\text {st }}$ of December 9 2019). Patients admitted with acute coronary syndrome (ACS) that had an ambiguous culprit lesion, or a borderline negative FFR on a possible culprit lesion, lesions located at the ostium of the right coronary artery (RCA), the left main stem or a graft and cases at the edge of a stent $(<5 \mathrm{~mm}$ from the edge of the

\section{Clinical endpoints}


1 The primary endpoint of the study was the incidence of lesion-oriented clinical events (LOCE) - defined

2 as the composite of cardiac death, target lesion related myocardial infarction (MI) or clinically indicated

3 target lesion revascularization (TLR). Cardiac death was defined as death caused from an acute MI,

4 sudden cardiac death, or death due to heart failure, while the diagnosis of MI was based on evidence of

5 myocardial necrosis (i.e., dynamic troponin rise) and supporting information derived from the clinical

6 presentation, electrocardiographic changes or the results of coronary angiography. ${ }^{14}$ Clinically indicated

$7 \quad$ TLR was performed in patients who had increased angina symptoms due to disease progression at repeat

8 coronary angiography - visually estimated by the interventional cardiologist - with or without evidence

9 of ischemia assessed by FFR or non-invasive imaging.

10 The classification of an event as target lesion or non-target lesion related was performed by two expert

11 analysts $(\mathrm{CVB}, \mathrm{AR})$ who reviewed the coronary angiography at the time of the event blindly to the 12 baseline demographics, 3D-QCA analysis and WSS estimations. MI or revascularisation was defined as 13 target lesion related when the event was attributed to significant disease progression in the lesion 14 defined by its proximal and distal edge at baseline angiography - that was assessed by FFR at baseline. 15 Any disagreement between experts was resolved by consensus.

16 Secondary endpoint of the study was the combined endpoint of target lesion related MI and/or 17 revascularisation.

\section{D-QCA reconstruction and WSS computation}

$203 \mathrm{D}-\mathrm{QCA}$ analysis was performed by an experienced analyst (VT) blindly to patient demographics and 21 clinical outcomes using a dedicated software (QAngio XA 3D RE - Medis Medical Imaging Systems) which assumes that the 3D lumen has elliptical cross sections. Reconstruction was performed for the main vessel and side branches with diameter $\geq 1 \mathrm{~mm}$ (Online Supplementary Materials). In the obtained 3D geometries, the lesion length, the \% area stenosis (AS) and the reference and minimum lumen area (MLA) were estimated. The 3D models were then processed with computational 26 fluid dynamic (CFD) techniques and the WSS distribution was estimated (Online Supplementary 27 Materials). The location of the lesions was identified in the processed models and the lesions were 28 divided in consecutive $3 \mathrm{~mm}$ segments. For each $3 \mathrm{~mm}$ segment, the WSS was extracted across the 
1 circumference and length of the segment and the mean value was calculated. For each lesion the lowest

2 and highest mean WSS value, estimated in the $3 \mathrm{~mm}$ segments of the lesion, were recorded and 3 corresponded to the "minimum" and "maximum" WSS of the lesion, respectively (Supplementary 4 Figure 1$) \cdot{ }^{13}$

5 The reproducibility of 3D-QCA analysis and WSS computation was tested using intraclass correlation 6 coefficient analysis in 20 patients; 3D-QCA and CFD analysis was performed twice by an expert analyst 7 within a 2-month interval and these data were used to examine the intra-observer variability. The inter8 observer variability was examined by comparing the estimations of the $1^{\text {st }}$ analyst with the estimations 9 of a $2^{\text {nd }}$ analyst.

11 Statistical analysis

12 The distribution of continuous variables was examined using the Kolmogorov-Smirnov test; a nonnormal distribution was found and therefore results were presented as median and inter-quartile range 14 (IQR). Categorical values were presented as absolute values and percentages. Comparison between

continuous variables were performed using the Mann Whitney U test, while categorical variables were compared using the chi-square or Fisher's exact test. Cox regression analysis was used to identify clinical, angiographic, 3D-QCA and WSS predictors associated with LOCE. Receiver operating characteristic (ROC) curve analysis was performed to identify amongst WSS variables the best predictor that was then entered into a multivariable model which included all the clinical, angiographic and 3DQCA predictors of LOCE. In case of collinear variables ( $\mathrm{R} \geq 0.5)$, only the variable with the highest area under the curve (AUC) in ROC curve analysis was entered into the model.

ROC curve analysis was also performed to identify the best cut-off for the 3D-QCA and WSS variables that were independently associated with LOCE. These cut-offs were used to classify lesions and patients in groups. Kaplan-Meier plots were used to display time to event at a lesion and patient level. In case of tandem lesions or patients with multiple lesions with a borderline negative FFR, the best lesion-level anatomical or physiological predictor of LOCE was used to define the most vulnerable lesion and this lesion characteristics were entered in the analysis. Due to the small number of patients $(n=7)$ with more than one lesion with a borderline negative FFR and the smaller number of patients $(n=1)$ that had a 
1 lesion which caused an event and a lesion that remained quiescent a clustering patient-level effect was

2 not added. The statistical analysis was performed using the SPSS Statistics 25 (IBM, Chicago, Ill., 3 USA); a p-value $<0.05$ was considered statistically significant.

4

\section{RESULTS}

6 Seven hundred thirteen patients were found to have at least one coronary lesion with a borderline 7 negative FFR (0.81-0.85), but only patients who had complete follow-up data $(n=548)$ were considered 8 for inclusion. Of these, 286 patients (293 lesions) were included in the final analysis as shown in Figure 9 1. The median age of the studied patients was 64.5 (55-71) years, most of them were suffering from a chronic coronary syndrome $(78.5 \%)$ at the time of index procedure and were treated with aspirin $(98.2 \%)$ and a statin $(97.2 \%)$.

During a median follow-up of 49.4 months, 37 LOCE were reported: 6 cardiac deaths, 9 target lesion related MI and 22 clinically indicated TLR. As it is shown in the Supplementary Table 1 the lesions causing events exhibited significant disease progression at the time of the event. Patients who experienced a LOCE were more likely to have a history of ACS compared to those that did not have LOCE (control group); otherwise, there were no differences between the two groups regarding their baseline demographics (Table 1). The differences in the baseline characteristics between patients who had a target lesion related MI or TLR and those who did not are shown in Supplementary Table 2.

\section{D-QCA analysis and WSS distribution}

Coronary reconstruction and blood flow simulation were successfully performed in all the studied lesions. An excellent intra- and inter-observer agreement was noted for the estimations of the two analysts (Online Supplementary Material).

As shown in Table 2, there was no difference in the location of the lesions that caused LOCE and those that remained quiescent. Conversely, lesions that caused LOCE had a smaller MLA and a larger AS, but there was no difference between the two groups in lesion length. In addition, the minimum WSS and the maximum WSS were higher in lesions that progressed and caused events than the lesions that were quiescent, while the coronary flow velocity was similar in the two groups. Similar findings were 
1 reported when analysis focused on lesions that caused MI or TLR during follow-up (Supplementary

2 Table 3).

3

4

5

6

\section{Predictors of LOCE and target lesion related MI or TLR: lesion level analysis}

Primary endpoint

In univariable Cox regression analysis one clinical variable (admission because of ACS at the time of the index procedure), two 3D-QCA (MLA and AS) and two CFD-derived variables (minimum WSS and maximum WSS) were predictors of LOCE (Table 3). The maximum WSS appeared to be the strongest haemodynamic predictor of LOCE - as this variable had the highest AUC in ROC analysis (0.72) - and was entered into the multivariable model. Multivariable Cox regression analysis demonstrated that AS and maximum WSS but not FFR were independently associated with LOCE (Table 3). Of note, these two variables were not collinear $(\mathrm{R}=0.478, \mathrm{p}=0.001)$.

The best AS and maximum WSS cut-off values that predicted LOCE in ROC curve analysis was $58.6 \%$ (sensitivity $81.1 \%$, specificity $61.3 \%$ ) and $7.69 \mathrm{~Pa}$ (sensitivity $78.4 \%$, specificity $55.1 \%$ ), respectively. As it is shown in Figure 2A, lesions with an increased AS ( $\geq 58.6 \%)$ that were exposed to high maximum WSS ( $\geq 7.69 \mathrm{~Pa})$ were at a higher risk of causing LOCE $(27.8 \%)$ than those that had increased AS and low WSS (12.8\%) or those that had a low AS and were exposed to high $(7.4 \%)$ or low WSS $(2.7 \%$, $\mathrm{p}<0.001)$.

\section{Secondary endpoint}

Similar results were reported for the secondary endpoint of target lesion related MI or TLR. History of previous CABG, AS, MLA and WSS but not FFR were associated with the secondary endpoint. AS and maximum WSS were the only independent predictors of target lesion related events (Table 3).

The best AS cut-off for predicting target lesion related MI or TLR was $58.6 \%$ (sensitivity $87.1 \%$, specificity $61.1 \%$ ), while the best cut-off for the maximum WSS was $8.65 \mathrm{~Pa}$ (sensitivity $74.2 \%$, specificity $63 \%$ ). These cut-off values were used to classify lesions in 4 groups. As shown in the KaplanMeier analysis, lesions with increased maximum WSS and AS were more likely to cause target lesion related MI or TLR than lesions with low AS and/or low WSS (p<0.001, Figure 2B). 
1 Patient level analysis for the primary and secondary endpoint showed similar findings to those reported

2 in the lesion level analysis (Online Supplementary Material).

3

4

5

6

7

8

9 10

\section{DISCUSSION}

In the present study we investigated, for the first time, the prognostic value of 3D-QCA-derived variables and WSS distribution in patients with a borderline negative FFR. We retrospectively processed angiographic data from 286 patients that had a lesion with FFR between 0.81 and 0.85 and found that: 1) these lesions were associated with an increased cardiovascular risk as the event rate was $12.9 \%$ at 4 year follow-up; 2) 3D-QCA-derived variables and in particular the MLA and AS provided useful prognostic information and identification of lesions that were likely to cause events and that 3) WSS distribution had an incremental prognostic value to 3D-QCA-derived variables enabling more accurate vulnerable plaque detection and risk stratification.

Several studies have demonstrated that the assessment of coronary physiology using FFR enables not only optimal treatment planning, but also identification of patients at risk. ${ }^{5,15,16} \mathrm{~A}$ pre-specified analysis of the FAME-2 (Fractional Flow Reserve versus Angiography for Multivessel Evaluation-2) study that included 607 patients treated conservatively showed that FFR is an independent predictor of major adverse cardiovascular events at 2-year follow-up. ${ }^{16}$ In this study, increased event rate was noted in lesions with FFR $\leq 0.80$; however, even in patients with non-ischemic lesions the FFR seemed to have a predictive value as patients with FFR between 0.81-0.85 had a higher event rate than those with FFR $>0.85$. Similar findings were reported by other studies that examined outcomes in patients with nonflow limiting stenoses showing that lesions with a borderline negative FFR are at risk of causing events. ${ }^{2-}$ ${ }^{4}$ A possible explanation of these observations comes from studies assessing the association between lesion haemodynamic severity and plaque morphology. ${ }^{17-19}$ Chen et al. in a study that included 323 lesions assessed by FFR and intravascular ultrasound imaging showed that there is a positive correlation between FFR and MLA and an inverse association between FFR and plaque burden. ${ }^{17}$ In addition, Tian et al. demonstrated that angiographic lesion severity was associated with plaque vulnerability assessed by combined intravascular ultrasound and optical coherence tomography imaging; more specifically, severely stenotic lesions (diameter stenosis $>70 \%$ ) were more likely to have a thin cap fibroatheroma 
1 phenotype, positive remodelling and increased plaque burden than lesions with a mild or moderate

2 stenosis on coronary angiography. ${ }^{18}$ These findings have also been confirmed by computed tomography

3 coronary angiography (CTCA) studies showing that there is a positive association between lesion

4 haemodynamic severity and high-risk plaque features..$^{19}$ Therefore, it can be speculated that the lesions

5 with a borderline negative FFR are more likely to have a high-risk phenotype (i.e., a thin or a thick cap

6 fibroatheroma), especially in patients admitted with ACS, and rapidly progress and cause events than

7 the lesions with a mild haemodynamic severity and higher FFR values.

8 Local haemodynamic forces and in particular WSS appear to regulate atherosclerotic disease 9 progression. ${ }^{6}$ Numerous CFD analyses in models reconstructed by intravascular imaging data have 10 provided mechanistic insights about the role of WSS on plaque formation and destabilisation and 11 highlighted its prognostic implications. ${ }^{7,8}{ }^{80}$ However, these reconstructions are time consuming and require intravascular imaging which is not commonly performed in daily practice. To address these limitations and bring WSS computation in the clinical arena 3D-QCA and CTCA-based modelling have been proposed. ${ }^{12,19}$ These simulations have a limited accuracy and do not enable precise evaluation of WSS distribution, especially in lesions with a complex anatomy or an eccentric obstruction where flow disturbances and high and low WSS co-exist. Therefore, these analyses focus on the estimation of the mean WSS value in a segment of interest instead of the local minimum or maximum value aiming to derive a prognostic marker and not to explore the interplay between plaque morphology and physiology and the role of WSS on plaque progression, destabilization and rupture; on the other hand, they are fast and appear to provide useful prognostic information.

A recent CFD analysis performed in 3D-QCA reconstructions that included 58 patients from the FAME2 study demonstrated that flow limiting stenoses (FFR $\leq 0.80)$ exposed to high WSS are more likely to cause MI than lesions exposed to low WSS. ${ }^{12}$ Similar findings were also reported in the EMERALD (Exploring the MEchanism of plaque Rupture in Acute coronary syndrome using coronary CT Angiography and computational fluid Dynamics) study where blood flow simulation in models reconstructed by CTCA showed that high WSS provided incremental prognostic information to plaque 27 characteristics and predicted more accurately lesions that caused MI. ${ }^{19}$ Nevertheless, these studies 28 included a small number of patients that mainly had flow limiting lesions $-49 \%$ of the lesions included 
1 in the EMERALD and all the lesions in the CFD analysis of the FAME-2 study had an FFR $\leq 0.80$ -

2 where revascularisation is indicated according to the current guidelines. ${ }^{1}$ Finally, a recent post-hoc 3 analysis of the IBIS-4 (Integrated Biomarkers Imaging Study-4) and PROSPECT (Providing Regional 4 Observations to Study Predictors of Events in the Coronary Tree) studies showed that WSS estimated 5 in 3D-QCA models have incremental value to plaque morphology in identifying amongst non-flow 6 limiting lesions with a vulnerable phenotype those that are likely to progress and cause major adverse 7 cardiovascular events..$^{13}$

8 The present study may constitute a paradigm shift in the search of the vulnerable plaque. We used FFR, 9 which today constitutes the gold standard for assessing lesion severity, and not intravascular imaging to identify non-flow limiting lesions that are at risk of causing events. Then, we processed models reconstructed from 3D-QCA with CFD techniques using a software that enabled fast blood flow simulation. We found that AS and WSS were independently associated with lesions prone to progress and cause events and that their combination enabled more accurate risk stratification. Although it would have been expected these variables to be collinear as WSS depends on lumen dimension, a weak correlation between WSS and AS was observed. This should be attributed to the fact that other factors such as inflow velocity, presence of bifurcation and the size of the side branch determine the flow through the lesion and consequently affect WSS. The WSS cut-off of $\geq 7.69 \mathrm{~Pa}$ found in our analysis is in line with experimental studies showing that WSS $>7 \mathrm{~Pa}$ has unfavourable effects on vessel wall biology. ${ }^{9}$ This value is higher than the cut-off of $4.71 \mathrm{~Pa}$ reported in the study of Kumar et al., a discrepancy that is likely to be due to the differences in the post-processing of the reconstructed models as they estimated the mean WSS in $5 \mathrm{~mm}$ segments while our analysis focused on $3 \mathrm{~mm}$ segments. ${ }^{12}$ Apart from the WSS, also 3D-QCA-derived variables and in particular the MLA and the AS also provided useful prognostic information, with the AS appearing an independent predictor in multivariable analysis. These findings are in line with the reported literature. ${ }^{16}$ The combination of AS and WSS enabled only more accurate identification of vulnerable lesions and had a positive predictive value of $27.8 \%$ that compares favourably with the findings of prospective intravascular imaging studies 27 of atherosclerosis and allowed detection of patients who are at risk of suffering a cardiovascular event. ${ }^{21-}$ $28{ }^{23}$ Considering the fact that FFR is routinely used in the clinical arena to assess lesion severity and that 
1 WSS computation is fast in 3D-QCA models, we believe that the present approach might be clinically

2 relevant in the future to detect vulnerable lesions and high-risk patients that will benefit from emerging

3 focal or systemic therapies of atherosclerosis ${ }^{24}$; however, these findings have to be confirmed in other 4 patient cohorts and ideally in prospective large-scale studies before advocating their broad use in clinical 5 practice.

\section{$7 \quad$ Limitations}

8 Although the present study is one of the largest analyses reported in the literature associating 9 haemodynamic variables with clinical events, it has limitations that should be acknowledged. First, its retrospective design has led to the exclusion of a large number of patients who had insufficient clinical or angiographic data or poor angiographic image quality, and this may introduce a selection bias. This also resulted in a small number of hard clinical endpoints and did not allow us to examine the value of the WSS in predicting cardiac death or MI. Nevertheless, it has to be acknowledged that aggressive atherosclerotic disease progression may have a similar pathophysiological pattern with ACS, as it has been recognised that not all the ruptured plaques cause MI but some of them tend to heal and progress fast causing angina symptoms. ${ }^{25}$ Moreover, the combined endpoint of our study is similar to the primary endpoint of all the reported and ongoing studies that also considered the clinically indicated TLR as a significant adverse cardiovascular event (PREVENT, NCT02316886; COMBINE OCT-FFR, NCT02989740). ${ }^{71-23,26}$ Additionally, we have included in this study patients admitted with chronic coronary syndrome and those with ACS; studies have showed significant differences in lesion morphology between these two populations which is likely to determine the implication of WSS on vessel pathology and clinical outcomes. ${ }^{27}$ To overcome this limitation the clinical presentation was included in the Cox regression analysis. It has to be stressed, however, that FFR is in both groups the standard invasive approach to assess lesion functional significance and the same cut-off of $>0.80$ is recommended to defer revascularization.

Furthermore, despite the strict exclusion criteria and the particular effort that was made to include only patients with high quality angiographic projections, often we processed suboptimal angiographic views with some foreshortening. A prospective study is likely to overcome these limitations and provide X- 
1 ray imaging data with excellent quality that will allow more accurate coronary reconstruction and

2 estimation of WSS distribution.

3 Finally, despite the approximations that were made in coronary artery modelling - using 3D-QCA

4 software that assumes the lumen has elliptical cross-sections - and in WSS estimation to expedite CFD

5 analysis, it has to be acknowledged that blood flow simulation remains time-consuming as this process

6 required approximately 20 minutes per vessel in our study. Nevertheless, future developments are

7 expected to further reduce the computational time to only few minutes allowing evaluation of WSS

8 distribution in real time while the patient is on the catheterisation laboratory. Recently, a software that

9 has been designed by Pie Medical Imaging (CAAS Workstation WSS, Pie Medical Imaging, Maastricht, 10 the Netherlands) that allows computation of WSS in 3D-QCA models within only few minutes and is 11 expected to broaden the applications of CFD in the clinical practice.

\section{Conclusions}

14 In this large-scale retrospective analysis, WSS distribution and 3D-QCA derived variables enabled 15 accurate detection of non-flow limiting lesions with a borderline negative FFR that are likely to progress and cause events and allowed identification of patients who are at risk of suffering LOCE. Prospective studies are needed to confirm these findings and developments in software design are required to expedite CFD analysis before this approach may be used to detect vulnerable lesions and patients who would benefit from novel focal or systemic therapies of atherosclerosis.

\section{Financial support}

25 VT, AR, RB, AM, DJ, AB, CVB are funded by the Barts NIHR Biomedical Research Centre. HS is 26 supported by research funds of the British Heart Foundation (Project Grant: PG/17/18/32883). CL is 27 funded by the UCL NIHR Biomedical Research Centre and a Wellcome/EPSRC Centre award $28[203145 Z / 16 / Z \&$ NS/A000050/1]. 


\section{Author contributions}

2 VT performed 3D-QCA and CFD analysis and wrote the first draft of the paper. CVB designed the study 3 and supervised data analysis. HS developed tools for CFD analysis and wrote the first draft of the paper.

4 RT supervised CFD analysis and revised the manuscript. RB, JZ, EH, KK and CDL collected the data 5 and together with $\mathrm{AR}, \mathrm{PK}, \mathrm{KBK}, \mathrm{AM}, \mathrm{DJ}, \mathrm{AL}, \mathrm{RR}, \mathrm{GVK} \mathrm{AB}$ and $\mathrm{CVB}$ reviewed the manuscript and 6 contributed to its content. All the authors have read and approved the final version of the manuscript. 7

\section{Acknowledgments}

9 None. 
[1] Neumann, FJ, Sousa-Uva, M, Ahlsson, A, et al., 2018 ESC/EACTS Guidelines on myocardial revascularization, Eur Heart J, 2019;40:87-165.

[2] Masrani Mehta, S, Depta, JP, Novak, E, et al., Association of Lower Fractional Flow

Reserve Values With Higher Risk of Adverse Cardiac Events for Lesions Deferred

Revascularization Among Patients With Acute Coronary Syndrome, J Am Heart Assoc, 2015;4:e002172.

[3] Adjedj, J, De Bruyne, B, Flore, V, et al., Significance of Intermediate Values of Fractional Flow Reserve in Patients With Coronary Artery Disease, Circulation, 2016;133:502508.

[4] Hakeem, A, Edupuganti, MM, Almomani, A, et al., Long-Term Prognosis of Deferred Acute Coronary Syndrome Lesions Based on Nonischemic Fractional Flow Reserve, J Am Coll Cardiol, 2016;68:1181-1191.

[5] Johnson, NP, Toth, GG, Lai, D, et al., Prognostic value of fractional flow reserve: linking physiologic severity to clinical outcomes, J Am Coll Cardiol, 2014;64:1641-1654.

[6] Thondapu, V, Bourantas, CV, Foin, N, et al., Biomechanical stress in coronary atherosclerosis: emerging insights from computational modelling, Eur Heart J, 2017;38:8192.

[7] Stone, PH, Saito, S, Takahashi, S, et al., Prediction of progression of coronary artery disease and clinical outcomes using vascular profiling of endothelial shear stress and arterial plaque characteristics: the PREDICTION Study, Circulation, 2012;126:172-181.

[8] Stone, PH, Maehara, A, Coskun, AU, et al., Role of Low Endothelial Shear Stress and Plaque Characteristics in the Prediction of Nonculprit Major Adverse Cardiac Events: The PROSPECT Study, JACC Cardiovasc Imaging, 2018;11:462-471.

[9] Gijsen, F, Katagiri, Y, Barlis, P, et al., Expert recommendations on the assessment of wall shear stress in human coronary arteries: existing methodologies, technical considerations, and clinical applications, Eur Heart J, 2019;40:3421-3433.

[10] Bourantas, CV, Tweddel, AC, Papafaklis, MI, et al., Comparison of quantitative coronary angiography with intracoronary ultrasound. Can quantitative coronary angiography accurately estimate the severity of a luminal stenosis?, Angiology, 2009;60:169-179.

[11] Bourantas, CV, Ramasamy, A, Karagiannis, A, et al., Angiographic derived endothelial shear stress: a new predictor of atherosclerotic disease progression, Eur Heart J Cardiovasc Imaging, 2019;20:314-322.

[12] Kumar, A, Thompson, EW, Lefieux, A, et al., High Coronary Shear Stress in Patients With Coronary Artery Disease Predicts Myocardial Infarction, J Am Coll Cardiol, 2018;72:1926-1935.

[13] Bourantas, CV, Zanchin, T, Torii, R, et al., Shear Stress Estimated by Quantitative Coronary Angiography Predicts Plaques Prone to Progress and Cause Events, JACC Cardiovasc Imaging, 2020.

[14] Hicks, KA, Tcheng, JE, Bozkurt, B, et al., 2014 ACC/AHA Key Data Elements and Definitions for Cardiovascular Endpoint Events in Clinical Trials: A Report of the American College of Cardiology/American Heart Association Task Force on Clinical Data Standards (Writing Committee to Develop Cardiovascular Endpoints Data Standards), J Am Coll Cardiol, 2015;66:403-469.

[15] Ahn, JM, Park, DW, Shin, ES, et al., Fractional Flow Reserve and Cardiac Events in Coronary Artery Disease: Data From a Prospective IRIS-FFR Registry (Interventional 
1 Cardiology Research Incooperation Society Fractional Flow Reserve), Circulation,

2 2017;135:2241-2251.

3 [16] Barbato, E, Toth, GG, Johnson, NP, et al., A Prospective Natural History Study of

4 Coronary Atherosclerosis Using Fractional Flow Reserve, J Am Coll Cardiol, 2016;68:2247-

52255.

6 [17] Chen, SL, Xu, B, Chen, JB, et al., Diagnostic accuracy of quantitative angiographic and

7 intravascular ultrasound parameters predicting the functional significance of single de novo

8 lesions, Int J Cardiol, 2013;168:1364-1369.

9 [18] Tian, J, Ren, X, Vergallo, R, et al., Distinct morphological features of ruptured culprit

10 plaque for acute coronary events compared to those with silent rupture and thin-cap

11 fibroatheroma: a combined optical coherence tomography and intravascular ultrasound

12 study, J Am Coll Cardiol, 2014;63:2209-2216.

13 [19] Lee, JM, Choi, G, Koo, BK, et al., Identification of High-Risk Plaques Destined to Cause

14 Acute Coronary Syndrome Using Coronary Computed Tomographic Angiography and

15 Computational Fluid Dynamics, JACC Cardiovasc Imaging, 2019;12:1032-1043.

16 [20] Bourantas, CV, Raber, L, Sakellarios, A, et al., Utility of Multimodality Intravascular

17 Imaging and the Local Hemodynamic Forces to Predict Atherosclerotic Disease Progression, 18 JACC Cardiovasc Imaging, 2019.

19 [21] Prati, F, Romagnoli, E, Gatto, L, et al., Relationship between coronary plaque 20 morphology of the left anterior descending artery and 12 months clinical outcome: the 21 CLIMA study, Eur Heart J, 2020;41:383-391.

22 [22] Stone, GW, Maehara, A, Lansky, AJ, et al., A prospective natural-history study of 23 coronary atherosclerosis, N Engl J Med, 2011;364:226-235.

24 [23] Waksman, R, Di Mario, C, Torguson, R, et al., Identification of patients and plaques 25 vulnerable to future coronary events with near-infrared spectroscopy intravascular 26 ultrasound imaging: a prospective, cohort study, Lancet, 2019;394:1629-1637.

27 [24] Bourantas, CV, Garcia-Garcia, HM, Torii, R, et al., Vulnerable plaque detection: an 28 unrealistic quest or a feasible objective with a clinical value?, Heart, 2016;102:581-589.

29 [25] Yamamoto, MH, Yamashita, K, Matsumura, M, et al., Serial 3-Vessel Optical 30 Coherence Tomography and Intravascular Ultrasound Analysis of Changing Morphologies 31 Associated With Lesion Progression in Patients With Stable Angina Pectoris, Circ Cardiovasc 32 Imaging, 2017;10.

33 [26] Erlinge, D, Maehara, A, Ben-Yehuda, O, et al., PROSPECT II: A Prospective Natural 34 History Study Using NIRS-IVUS Imaging in Patients with Acute Myocardial Infarction, In, TCT, 352020.

36 [27] Kato, K, Yonetsu, T, Kim, SJ, et al., Nonculprit plaques in patients with acute coronary 37 syndromes have more vulnerable features compared with those with non-acute coronary 38 syndromes: a 3-vessel optical coherence tomography study, Circ Cardiovasc Imaging, 39 2012;5:433-440.

40 
TABLES

2 Table 1. Baseline demographics of the patients who suffered a LOCE and of the control group.

\begin{tabular}{l|cccc} 
& $\begin{array}{c}\text { Studied patients } \\
(\mathbf{n = 2 8 6})\end{array}$ & $\begin{array}{c}\text { LOCE group } \\
(\mathbf{n = 3 7})\end{array}$ & $\begin{array}{c}\text { Control group } \\
(\mathbf{n = 2 4 9})\end{array}$ & P \\
\hline Clinical characteristics & & & & \\
\hline Age & $64.5(55-71)$ & $62(54-71)$ & $65(56-71)$ & 0.414 \\
Male gender & $238(83.2)$ & $33(89.2)$ & $205(82.3)$ & 0.355 \\
Family history of CAD & $93(33.7)$ & $8(22.2)$ & $85(35.4)$ & 0.118 \\
ACS presentation & $61(21.5)$ & $13(35.1)$ & $48(19.4)$ & 0.030
\end{tabular}

\begin{tabular}{lcccc}
\hline Co-morbidities & & & & \\
\hline Hypertension & $190(71.4)$ & $26(70.3)$ & $164(71.6)$ & 0.867 \\
Hypercholesterolemia & $194(70.8)$ & $30(81.1)$ & $164(69.2)$ & 0.139 \\
Diabetes mellitus & $82(29.3)$ & $14(37.8)$ & $68(28)$ & 0.220 \\
History of smoking & $125(44.2)$ & $17(47.2)$ & $108(43.7)$ & 0.693 \\
Reduced LVEF $^{\mathrm{b}}$ & $32(14.4)$ & $6(22.2)$ & $26(13.3)$ & 0.218 \\
CKD $^{\mathrm{c}}$ & $40(14.9)$ & $9(25)$ & $31(13.4)$ & 0.068 \\
Previous MI & $83(29.3)$ & $11(29.7)$ & $72(29.3)$ & 0.954 \\
Previous PCI & $97(34.3)$ & $14(37.8)$ & $83(33.7)$ & 0.624 \\
Previous CABG & $5(1.8 \%)$ & $2(5.4)$ & $3(1.2)$ & 0.129
\end{tabular}

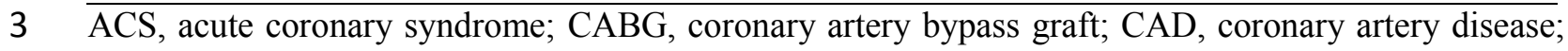

4 CKD, chronic kidney disease; LVEF, left ventricular ejection fraction; LOCE, lesion-oriented clinical

5 events; MI, myocardial infarction; PCI, percutaneous coronary intervention.

$6 \quad{ }^{\text {a }}$ History of smoking, defined as current or previous smoker.

$7 \quad{ }^{\mathrm{b}}$ Reduced LVEF was defined as ejection fraction $<50 \%$.

$8 \quad{ }^{\mathrm{c}} \mathrm{CKD}$ defined as estimated glomerular filtration rate $<60 \mathrm{~mL} / \mathrm{min} / 1.73 \mathrm{~m}^{2}$. 
1 Table 2. Angiographic, 3D-QCA and CFD-derived variables of lesions that caused and did not cause

2 LOCE.

\begin{tabular}{|c|c|c|c|c|}
\hline & $\begin{array}{l}\text { Studied lesions } \\
\qquad(\mathrm{n}=\mathbf{2 9 3})\end{array}$ & $\begin{array}{l}\text { LOCE group } \\
\qquad(\mathbf{n}=\mathbf{3 7})\end{array}$ & $\begin{array}{l}\text { Control group } \\
\qquad(\mathrm{n}=\mathbf{2 5 6})\end{array}$ & $\mathbf{P}$ \\
\hline \multicolumn{5}{|l|}{ Angiographic variables } \\
\hline Studied vessel & & & & 0.183 \\
\hline - LAD & $224(76.5)$ & $28(75.7)$ & $196(76.6)$ & \\
\hline$-\quad \mathrm{LCx}$ & $27(9.2)$ & $6(16.2)$ & $21(8.2)$ & \\
\hline - $\mathrm{RCA}$ & $42(14.3)$ & $3(8.1)$ & $39(15.2)$ & \\
\hline Lesion location & & & & 0.238 \\
\hline - Proximal vessel & $94(32.1)$ & $15(40.5)$ & $79(30.9)$ & \\
\hline - Mid-distal vessel & $199(67.9)$ & $22(59.5)$ & $177(69.1)$ & \\
\hline FFR value & $0.84(0.82-0.85)$ & $0.83(0.82-0.84)$ & $0.84(0.82-0.85)$ & 0.311 \\
\hline Coronary flow velocity $(\mathrm{mm} / \mathrm{sec})$ & $137(120.2-154.3)$ & $142.1(129.6-160.5)$ & $136.9(120-154.2)$ & 0.128 \\
\hline
\end{tabular}

\begin{tabular}{lllll}
\hline 3D-QCA variables & & & \\
\hline
\end{tabular}

CFD-derived variables
$\begin{array}{lcccc} \\ \text { Minimum WSS }(\mathrm{Pa}) & 1.49(1.04-2.19) & 1.92(1.28-2.37) & 1.48(1.01-2.08) & 0.017 \\ \text { Maximum WSS }(\mathrm{Pa}) & 7.62(5.66-10.92) & 10.72(7.86-15.14) & 7.35(5.54-10.22) & <0.001\end{array}$

3 3D-QCA, three-dimensional quantitative coronary angiography; AS, area stenosis; CFD, computational fluid

4 dynamic; FFR, fractional flow reserve; LAD, left anterior descending artery; LCx, left circumflex artery;

5 LOCE, lesion-oriented clinical events; MLA, minimum lumen area; RCA, right coronary artery; WSS, wall

6 shear stress. 
1 Table 3. Lesion level univariable and multivariable predictors of LOCE and target lesion related MI

2 or TLR.

\begin{tabular}{lcccc}
\hline & \multicolumn{2}{c}{ Univariable analysis } & \multicolumn{2}{c}{ Multivariable analysis $^{\mathbf{a}}$} \\
Variables & HR (95\% CI) & P & HR (95\% CI) & P \\
\hline LOCE & & & & \\
\hline ACS presentation & $1.98(1.01-3.88)$ & 0.048 & $1.69(0.86-3.37)$ & 0.131 \\
MLA (per 1mm² increase) & $0.55(0.33-0.90)$ & 0.018 & $1.02(0.70-1.48)$ & 0.918 \\
AS (per 1\% increase) & $1.09(1.05-1.12)$ & $<0.001$ & $1.06(1.03-1.10)$ & 0.001 \\
Minimum WSS (per 1Pa increase) & $1.47(1.13-1.92)$ & 0.005 & - & - \\
Maximum WSS (per 1Pa increase) & $1.12(1.07-1.17)$ & $<0.001$ & $1.08(1.02-1.14)$ & 0.012
\end{tabular}

Target lesion related MI or TLR

\begin{tabular}{lcccc}
\hline Previous CABG & $4.77(1.14-20.01)$ & 0.033 & $2.32(0.52-10.31)$ & 0.270 \\
MLA (per 1 $\mathrm{mm}^{2}$ increase) & $0.48(0.27-0.84)$ & 0.011 & $1.04(0.64-1.68)$ & 0.875 \\
AS (per 1\% increase) & $1.11(1.07-1.15)$ & $<0.001$ & $1.08(1.04-1.13)$ & $<0.001$ \\
Minimum WSS (per 1Pa increase) & $1.50(1.13-1.99)$ & 0.005 & - & - \\
Maximum WSS (per 1Pa increase) & $1.13(1.08-1.18)$ & $<0.001$ & $1.08(1.01-1.15)$ & 0.026
\end{tabular}

3 ACS, acute coronary syndrome; AS, area stenosis; CABG, coronary artery bypass graft; CI, confidence 4 interval; HR, hazard ratio; LOCE, lesion-oriented clinical events; MI, myocardial infarction; MLA, minimum 5 lumen area; TLR, target lesion revascularization; WSS, wall shear stress.

6 a'Maximum WSS was preferred to minimum WSS and entered into the multivariable model as it had the 7 highest area under the curve in the receiver-operating characteristics curve analyses performed for both 8 LOCE $\left(\mathrm{AUC}_{\min W S S}=0.62, \mathrm{P}=0.017 ; \mathrm{AUC}_{\operatorname{maxWSS}}=0.72, \mathrm{P}<0.001\right)$ and target lesion related $\mathrm{MI}$ or TLR $9 \quad\left(\mathrm{AUC}_{\min W S S}=0.61, \mathrm{P}=0.038 ; \mathrm{AUC}_{\operatorname{maxWSS}}=0.73, \mathrm{P}<0.001\right)$. 


\section{FIGURE LEGENDS}

2 Figure 1. Flowchart of the patients and lesions included in the present analysis.

3 3D-QCA, three-dimensional quantitative coronary angiography; BHC, Barts Heart Centre, London;

4 CTC, Cardiothoracic Centre, Basildon; FFR, fractional flow reserve; LMS, left main stem; LOCE, 5 lesion-oriented clinical events; RFH, Royal Free Hospital, London.

6 Figure 2. Kaplan-Meier curves display time to LOCE (A) and target lesion related MI or TLR (B) at a 7 lesion level analysis.

8 AS, area of stenosis; LOCE, lesion-oriented clinical events; MI, myocardial infarction; TLR, target 9 lesion revascularization; WSS, wall shear stress.

10 Graphical abstract. 3D-QCA modelling and WSS distribution enable more accurate risk stratification 11 and prediction of LOCE at 4-year follow-up.

12 3D-QCA, three-dimensional quantitative coronary angiography; AS, area of stenosis; CI, confidence 13 interval; HR, hazard ratio; LOCE, lesion-oriented clinical events; WSS, wall shear stress. 


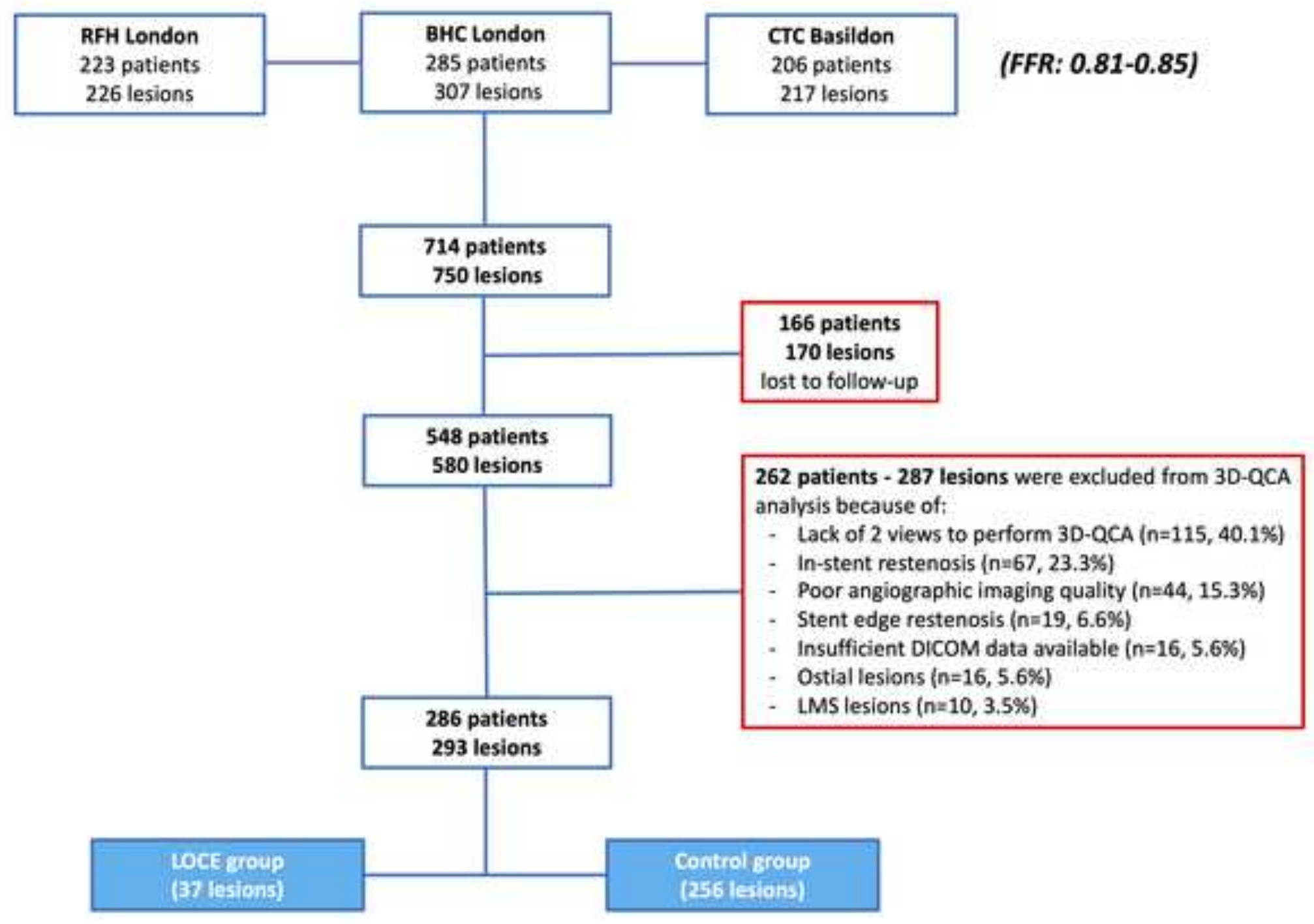

170 lesions

lost to follow-up

548 patients

analysis because of:

Lack of 2 views to perform 3D-QCA ( $n=115,40.1 \%$ )

Stent edge restenosis ( $n=19,6.6 \%)$

Insufficient DICOM data available $(n=16,5.6 \%)$

Ostial lesions $(n=16,5.6 \%)$

286 patients

LMS lesions ( $n=10,3.5 \%$ ) 

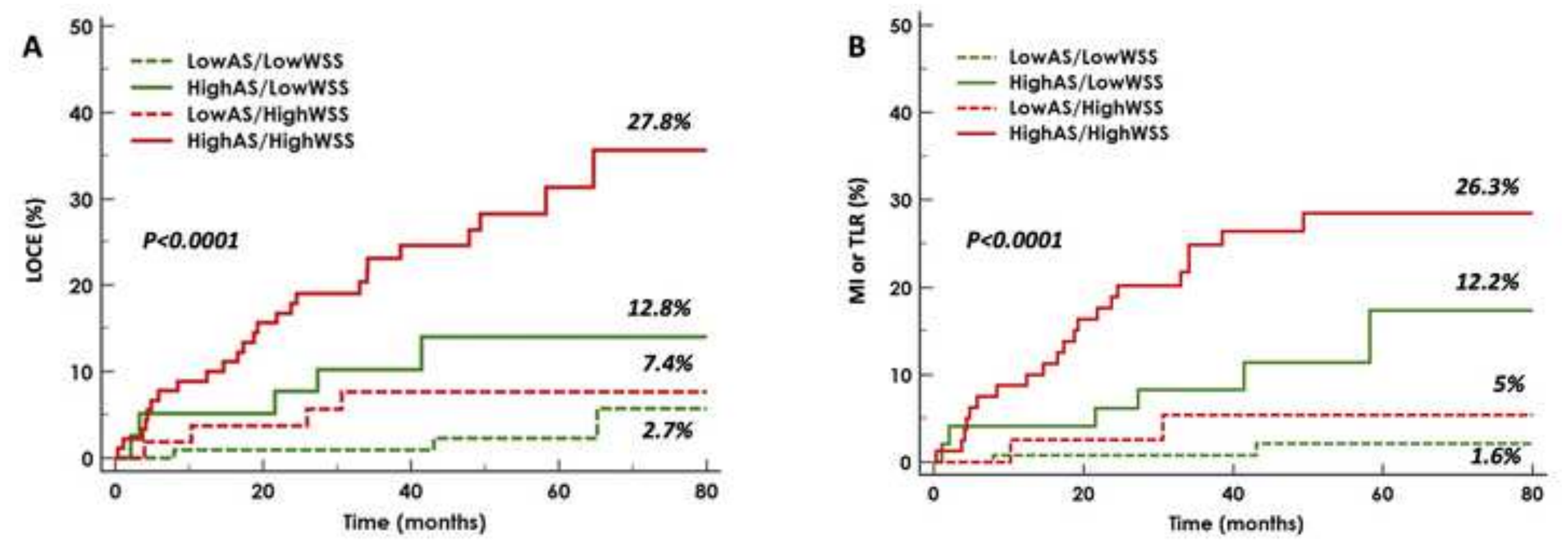